\title{
Effect of DJ-1 overexpression on the proliferation, apoptosis, invasion and migration of laryngeal squamous cell carcinoma SNU-46 cells through PI3K/AKT/mTOR
}

\author{
BIN WANG $^{1 *}$, HAO QIN ${ }^{2 *}$, YUEJIAN WANG ${ }^{2 *}$, WEIXIONG CHEN ${ }^{2}$, JIE LUO $^{1}$, \\ XIAOLIN ZHU ${ }^{1}$, WEIPING WEN ${ }^{1}$ and WENBIN LEI ${ }^{1}$
}

\begin{abstract}
${ }^{1}$ Otorhinolaryngology Hospital, Otorhinolaryngology Institute, The First Affiliated Hospital, Sun Yat-sen University, National Key Department of Otorhinolaryngology of People's Republic of China, Guangzhou, Guangdong 510080;

${ }^{2}$ Department of Otolaryngology, The First People's Hospital of Foshan, Foshan, Guangdong 528000, P.R. China
\end{abstract}

Received February 24, 2014; Accepted May 20, 2014

DOI: $10.3892 /$ or.2014.3286

\begin{abstract}
The aim of the present study was to explore the effect of DJ-1-mediated PI3K/AKT/mTOR pathway on the proliferation, apoptosis, invasion, migration and other tumor biological characteristics of laryngeal squamous cell SNU-46, through stable transfection and overexpression of the DJ-1 gene. Retrovirus carrying DJ-1 gene was used to stabilize transfected human laryngeal squamous carcinoma SNU-46 cell line, and monoclonal cell line of stably overexpressed DJ-1 protein was screened out by G418. DJ-1 protein expression was determined by western blotting, and changes of p-AKT, p-mTOR and PTEN protein content were detected, followed by the detection of changes in proliferation, apoptosis, invasion, migration and other tumor biological characteristics of laryngeal squamous carcinoma cell line with stably transfected DJ-1 protein overexpression by flow cytometry, CCK-8 method and Transwell. We successfully constructed a laryngeal squamous carcinoma cell line of stably overexpressed DJ-1 protein and termed it SNU-46-DJ-1. After overexpression of DJ-1 protein, the levels of PTEN expression in laryngeal squamous cell SNU-46 decreased and p-AKT and p-mTOR protein expression levels increased. Compared to the untreated SNU-46 cells, the proliferation rate of SNU-46-DJ-1 cells increased $(0.834 \pm 0.336$ vs. $0.676 \pm 0.112 ; \mathrm{p}<0.001)$; invasiveness was enhanced $(165.7 \pm 13.6$ vs. $100.0 \pm 17.4 ; p=0.001)$, the migration ability was enhanced (207.3 \pm 13.1 vs. $175.3 \pm 13.3$;
\end{abstract}

Correspondence to: Dr Wenbin Lei or Dr Weiping Wen, Otorhinolaryngology Hospital, Otorhinolaryngology Institute, The First Affiliated Hospital, Sun Yat-sen University, National Key Department of Otorhinolaryngology of People's Republic of China, 58 Zhongshan 2nd Road, Guangzhou, Guangdong 510080, P.R. China

E-mail: leiwb@mail.sysu.edu.cn

E-mail: wenwp@mail.sysu.edu.cn

${ }^{*}$ Contributed equally

Key words: stable transfection, DJ-1, PTEN, PI3K/AKT/mTOR signaling pathway, SNU-46 cell line, laryngeal squamous carcinoma $\mathrm{p}=0.036)$, and the apoptosis rate decreased $(3.533 \pm 5.167$ vs. $16.397 \pm 5.447 \%$; $\mathrm{p}=0.019$ ). The overexpression of DJ-1 protein in laryngeal squamous carcinoma SNU-46 cells can accelerate proliferation rate, increase the invasion and migration capacity, and reduce apoptosis, by activating the PI3K/AKT/ mTOR pathway.

\section{Introduction}

DJ-1 protein was discovered in 1997 by Nagakubo et al; it is widely expressed in various human tissues, with high expression levels in testis, kidney, pancreas, heart and skeletal muscle (1). The total length of the human DJ-1 gene is $24 \mathrm{ku}$, including 8 exons of which the first two do not encode proteins. The mRNA of DJ-1 gene is $570 \mathrm{bp}$, and encoding a DJ-1 protein containing 189 amino acids, with a molecular weight of $20 \mathrm{kDa}$. The DJ-1 gene is highly conserved in evolution, the homology of human and monkey DJ-1 gene is up to $98 \%$ and the homology to mouse is $91 \%(2,3)$. Formation, positive regulation of androgen receptor and the fertilization process, participation in the regulation of RNA binding complex (4), and anti-oxidation, inhibition of apoptosis (5). Previous studies on the DJ-1 gene were mostly on Parkinson's disease (PD)-related research, and they found that its mutations are related to human early-onset PD. However, some studies have found that the DJ-1 gene is closely related to tumor as well. Several studies found that DJ-1 protein overexpression is present in a variety of tumors such as breast cancer, non-small cell lung cancer, esophageal squamous cell carcinoma and prostate cancer, and this overexpression is related to proliferation, metastasis and poor prognosis of malignant tumors (6-10). Currently, carcinogenic DJ-1 gene downstream molecular signaling network and transduction mechanism remain unclear, and the role of the DJ-1 gene in the tumor formation and growth process is also unclear. Kim et al found that DJ-1 expression in breast cancer specimens was negatively correlated with PTEN, while positively correlated with PKB/Akt (6). Studies have also indicated that the DJ-1 gene can promote the PI3K/AKT/ mTOR pathway by regulating PTEN genes, thereby enabling increased expression of a variety of detoxifying enzymes to 
improve the cell viability and promote cell proliferation (11). Davidson et al detected PTEN protein expression levels of cancer tissues of nearly 300 cases of ovarian cancer patients with immunohistochemistry, and analyzed DJ-1 mRNA levels with RT-PCR. The results suggested that the levels of DJ-1 and PTEN were not correlated (12). Therefore, whether DJ-1 is involved in regulation of PTEN remains to be confirmed with further research. In the present study, retrovirus was used for stable transfection of DJ-1 gene to laryngeal squamous carcinoma cell line SNU-46, and the cell line was screened out to compare the changes in tumor biological characteristics (proliferation, apoptosis, invasion and migration) with untreated laryngeal cancer cell lines. In the present study, we preliminarily studied the effect of DJ-1 gene overexpression on laryngeal cancer cell line SNU-46 biological activities, $\mathrm{PI} 3 \mathrm{~K} / \mathrm{AKT} / \mathrm{mTOR}$ pathway and PTEN protein expression.

\section{Materials and methods}

\section{Materials}

Cell line and culture. Human laryngeal squamous cell carcinoma SNU-46 cells were cultured in RPMI-1640 medium containing $10 \%$ fetal bovine serum (FBS; HyClone) and incubated in a $37^{\circ} \mathrm{C}, 5 \% \mathrm{CO}_{2}$ humidified incubator. GP2-293 packaging cells were cultured in high glucose Dulbecco's modified Eagle's medium (DMEM) containing 10\% FBS.

Biomaterials. pCDNA3.1(+)-DJ-1 plasmid carrying DJ-1 gene coding sequence was stored in our laboratory. pLNCX2 plasmid, pVSV-G plasmid and GP2-293 packaging cells were kindly donated by Dr Lei Jinju from the Sun Yat-sen University Cancer Center (SYSUCC). Restriction endonucleases NotI and XhoI were purchased from Fermentas (USA), DJ-1 primers were purchased from Guangzhou RiboBio Co., DJ-1 monoclonal antibody was purchased from Abcam Co., GAPDH monoclonal antibody was purchased from BioWorld Co., HRP-labeled goat anti-mouse monoclonal antibody was purchased from Santa Cruz Co., AKT, phospho-Akt (Ser473), mTOR, phospho-mTOR (Ser2448) and PTEN antibody were purchased from Cell Signaling Technology, Inc.

\section{Methods}

Vector construction. Using PCR method, according to the DJ-1 gene sequence information and planned inserting restriction endonuclease gene site sequence, we designed the coding region amplification primers. pCDNA3.1(+)-DJ-1 plasmid was used as template to amplify its coding sequence (CDS) by PCR. The gene sequence and vector sequence were analyzed. Two restriction endonucleases NotI and XhoI may be used to clone destination gene fragment into the vector. Designed primers were: F, 5'CCGCTCGAGACCATGGCTTC CAAAAGAGCTCTGGTCAT3' and R, 5' ATTTGCGGCCG CCTAGTCTTTAAGAACAAGTGGAGCCTTCA3' (primer synthesis, Shanghai Sangon Co., Ltd.). The PCR products were purified by gel electrophoresis and recovered for plasmid splicing. Gel extraction kit was the product of Qiagen Co. (USA). Restriction endonucleases NotI and XhoI were used for double digestion of the above PCR product and pLNCX2 plasmids, followed by agarose gel electrophoresis, purification and recovery of the digested products. DNA Ligation kit (Takara) was used for connection. The connected plasmids were used to transform DH-5 $\alpha$ competent $E$. coli, followed by expanding culture, extraction and sequencing detection.

Transfection. The transfection was performed with the Lipofectamine 2000 Reagent (Invitrogen, Carlsbad, CA, USA), according to the manufacturer's protocol.

Retroviral packaging. The day before transfection, GP2-293 cells were inoculated in a $10 \mathrm{~cm}$ petri dish. High glucose DMEM medium containing $10 \%$ fetal calf serum was used for culture and transfection began at $50 \%$ cell fusion. Six micrograms of each pVSV-G and pLNCX2-DJ-1 plasmid was added to $1.5 \mathrm{ml} \mathrm{Opti-MEM} \mathrm{serum-free} \mathrm{medium,} \mathrm{and} \mathrm{then} 20 \mu \mathrm{l}$ Lipofectamine 2000 liposome transfection reagent was added to $1.5 \mathrm{ml}$ Opti-MEM serum-free medium. The two liquids were placed at room temperature for $5 \mathrm{~min}$, and then gently mixed together. The mixture was stored for $20 \mathrm{~min}$ and was added to GP2-293 cells. Six hours later, the medium was changed and $10 \mathrm{ml}$ fresh high glucose DMEM medium containing 10\% FBS was added. After 48 h, GP2-293 cell culture supernatant in the $10 \mathrm{~cm}$ petri dish was drawn and filtered through $0.45 \mu \mathrm{m}$ filter to remove cell debris and other impurities, and it was stored at $-80^{\circ} \mathrm{C}$. The same method was used to transfect pVSV-G and pLNCX2 empty vector plasmid to obtain empty vector virus of the control group, which was stored at $-80^{\circ} \mathrm{C}$.

Retroviral infection of SNU-46 cells. Twenty four hours before infection, the SNU-46 cells were inoculated in $10 \mathrm{~cm}$ petri dishes, with inoculation density of $\sim 50 \%$. Polybrene $(8 \mu \mathrm{g} / \mathrm{ml})$ was added to $5 \mathrm{ml}$ virus-containing supernatant which was added to SNU-46 cells. After $6 \mathrm{~h}$, the culture medium and virus-containing supernatant in SNU-46 cells were discarded. RPMI-1640 medium $(5 \mathrm{ml})$ and $5 \mathrm{ml}$ viral-containing supernatant $(8 \mu \mathrm{g} / \mathrm{ml}$ of polybrene added) were added again. After $6 \mathrm{~h}$, RPMI-1640 medium containing 10\% FBS was replaced. The above method was applied to the infection of SNU-46 cells with retrovirus carrying DJ-1 gene and empty vector virus of the control group.

Stable cell line selection. Cells were cultured continuously at $37^{\circ} \mathrm{C}$ for $48 \mathrm{~h}$. Antibiotic G418 $(200 \mu \mathrm{g} / \mathrm{ml})$ was initially used to screen the positive clones. After 10-12 days, cells were maintained continuously in the medium with G418 $(100 \mu \mathrm{g} / \mathrm{ml})$, until the cell colony was macroscopic. The surviving cells from G418 screening were prepared to cell suspensions. The cells were counted and diluted with cell culture medium to $1 / 10 \mu \mathrm{l}$. G418 screening medium prepared according to the best screening concentration was added to a 96-well plate, with $150 \mu \mathrm{l} /$ well, then cell suspension was added, with $10 \mu \mathrm{l} /$ hole. Twenty-four hours later, the plate was observed under a microscope. The well which had only one living cell was selected and labelled. Standard RPMI-1640 medium containing $15 \%$ FBS medium was replaced once every two days. When single cell was proliferated to $\sim 100$ cells, the cells in the wells were moved to a 48-well plate for continued culture after digestion. After proliferation, cells were moved to a 24-well plate, and so on, so cells were cultured progressively to obtain G418-resistant cell lines after several successful and stable transfections. 
CCK-8 assay. Cell Counting Kit-8 from Biyuntian Company was used in the experiment. Cell proliferation in each group was detected at 24, 48 and $72 \mathrm{~h}$, respectively, after cells were inoculated. The inoculated cells were suspended in 96-well plates, with $100 \mu \mathrm{l} /$ well, and each well contained 2,000 cells. The plates were pre-cultured in an incubator $\left(37^{\circ} \mathrm{C}, 5 \% \mathrm{CO}_{2}\right.$ overnight stable condition). During the experiment to determine the number of cells, $10 \mu \mathrm{l}$ CCK- 8 solution was added to each well, and the plate was incubated for $1.5 \mathrm{~h}$. The absorbance at $450 \mathrm{~nm}$ (OD) was measured by a microplate reader, and a cell growth curve was drawn with time as the abscissa and absorbance values as the vertical.

In vitro migration and invasion assays. For the Transwell migration or invasion assays, cells in $0.2 \mathrm{ml} \mathrm{RPMI-1640}$ without FBS were placed on the top chamber of each insert (BD Biosciences, Franklin Lakes, NJ, USA) with or without $40 \mu \mathrm{l}$ of $1 \mathrm{mg} / \mathrm{ml}$ Matrigel. The lower chamber was filled with $600 \mu 1$ of RPMI-1640 medium with $10 \%$ FBS to act as the nutritional attractant. Twenty-four hours later, the migrant cells that had attached to the lower surface were fixed with $20 \%$ methanol and stained for 20 min with crystal violet. The membranes were then carved and embedded under cover slips with the cells on the top. Cells in three different fields of view at x100 magnification were counted and expressed as the average number of cells per field of view. All assays were performed in triplicate.

Real-time RT-PCR. Real-time RT-PCR was performed to detect the relative level of DJ-1 transcription and mature miRNA. Briefly, complement DNA (cDNA) was generated through reverse transcription using M-MLV reverse transcriptase (Promega) with $1 \mu \mathrm{g}$ of total RNA. This cDNA product was used to amplify the DJ-1 gene. The PCR conditions were: $95^{\circ} \mathrm{C}$ for $5 \mathrm{~min}$, followed by 40 cycles of $95^{\circ} \mathrm{C}$ for $15 \mathrm{sec}, 60^{\circ} \mathrm{C}$ for $15 \mathrm{sec}$ and $72^{\circ} \mathrm{C}$ for $32 \mathrm{sec}$.

Western blot analysis. The cells were lysed with RIPA lysis buffer and the proteins were harvested. All proteins were resolved on a $10 \%$ SDS denatured polyacrylamide gel and then transferred onto a nitrocellulose membrane. The membranes were incubated overnight at $4^{\circ} \mathrm{C}$ with mouse monoclonal to PARK7/DJ1 (Abcam, USA) and rabbit monoclonal anti-human GAPDH, PTEN, AKT/phospho-Akt (Ser473), mTOR/phospho-mTOR (Ser2448) (Cell Signaling Technology, Inc.). Goat anti-mouse IgG-HRP (1:1,000; Jackson Co.) and goat anti-rabbit IgG-HRP (1:500; Santa Cruz Inc.) were used for secondary antibody and DAB coloring.

Flow cytometry. Apoptosis detection kit from Nanjing Kaiji Co. was used in the experiment. Cells were collected with EDTA-free trypsin and washed twice with PBS, followed by centrifugation $(2,000 \mathrm{rpm})$ for $5 \mathrm{~min}$. Binding buffer $(500 \mu \mathrm{l})$ was added for cell suspension. Annexin V-EGFP (5 $\mu 1)$ was added and mixed well, followed by addition of $5 \mu 1$ propidium iodide and mixed well. The mixture was reacted at room temperature for 5-15 min in a dark environment, followed by detection with flow cytometry, excitation wavelength $\mathrm{Ex}, 488 \mathrm{~nm}$; emission wavelength Em, $530 \mathrm{~nm}$. Green fluorescence of Annexin V-EGFP was detected with FITC channel

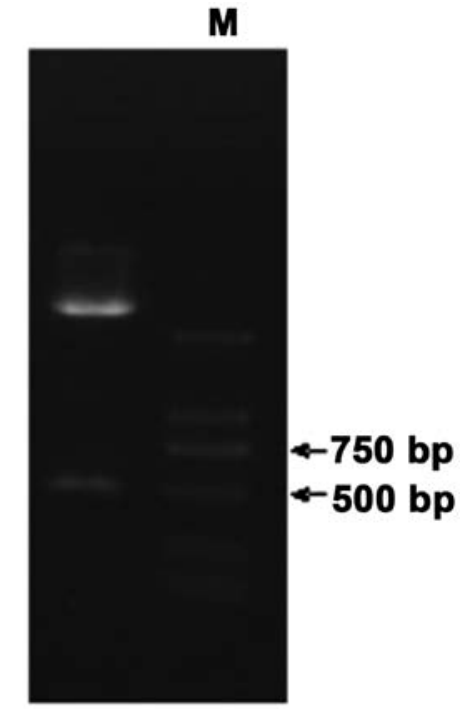

Figure 1. Recombinant plasmid pLNCX2-DJ-1 double digestion.

(FL1); PI red fluorescence was detected by PI channel (FL2 or FL3). Fluorescence compensation adjustment; normal untreated cells were used as control for fluorescence compensation adjustment to remove spectra overlap and set the cross gate position.

Statistical analysis. All measurement data are expressed as the means \pm SD. Differences among groups were analyzed by one-way ANOVA and Student-Newman-Keuls (SNK) q-test using SPSS 12.0 software for Windows. Statistical significance was defined as having a p-value $<0.05$.

\section{Results}

Double enzyme digestion of the recombinant plasmid $p L N C X 2-D J-1$. The recombinant plasmid pLNCX2-DJ-1 was transformed into competent $E$. coli, and then the plasmid was extracted after shaking bacteria. NotI, XhoI double digestion was performed on the exacted plasmid. The digestion result was detected with $1 \%$ agarose gel electrophoresis. There was clearly visible strip for the double digestion products near $600 \mathrm{bp}$ (Fig. 1). Forward sequencing was carried out for the recombinant plasmid pLNCX2-DJ-1 according to pLNCX2 carrier up and downstream primer sequences. BLAST alignment was performed for the sequencing results (Beijing Liuhehua Biotechnology) with DJ-1 sequence in NCBI and matching degree was $100 \%$.

Determination of G418 screening concentration. After 10 concentration gradient tests of G418 in $100-1,000 \mu \mathrm{g} / \mathrm{ml}$, the minimum G418 concentration in which cells died within 10 days was $100 \mu \mathrm{g} / \mathrm{ml}$. Therefore, the optimum concentration of G418 screening was $100 \mu \mathrm{g} / \mathrm{ml}$.

Screening of laryngeal carcinoma cell lines of stably transfected DJ-1 protein overexpression. SNU-46 cells infected with the virus were screened in a normal medium without G418. As shown in Fig. 2, cells infected with retroviral that did not obtain stable transfection gradually died without G418 resistance, and 


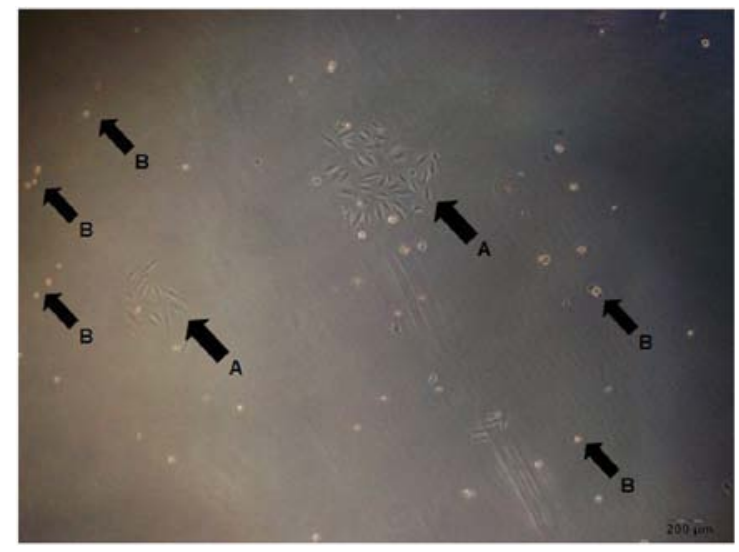

Figure 2. SNU-46 cells with successful stable transfection obtain G418 resistance. (A) SNU-46 cells with successful stable transfection obtained G418 resistance and normal growth; (B) SNU-46 cells without successful stable transfection did not obtain $\mathrm{G} 418$ resistance; nuclear condensation or cleavage appeared and cells died.

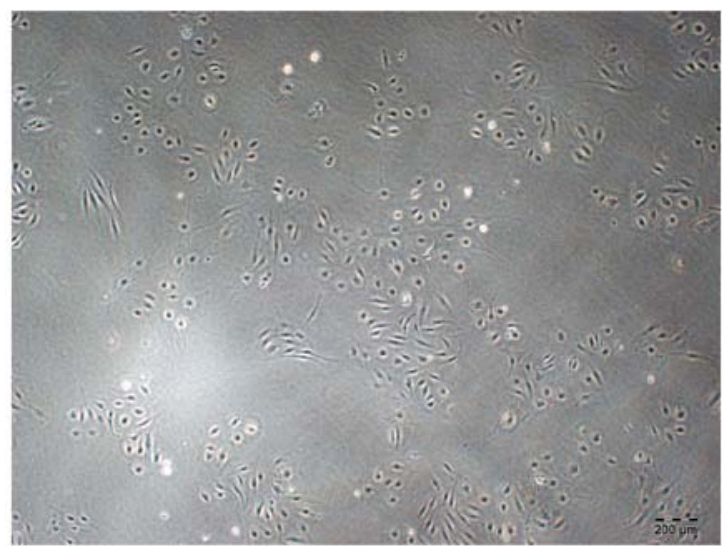

Figure 3. SNU-46-D1 cells

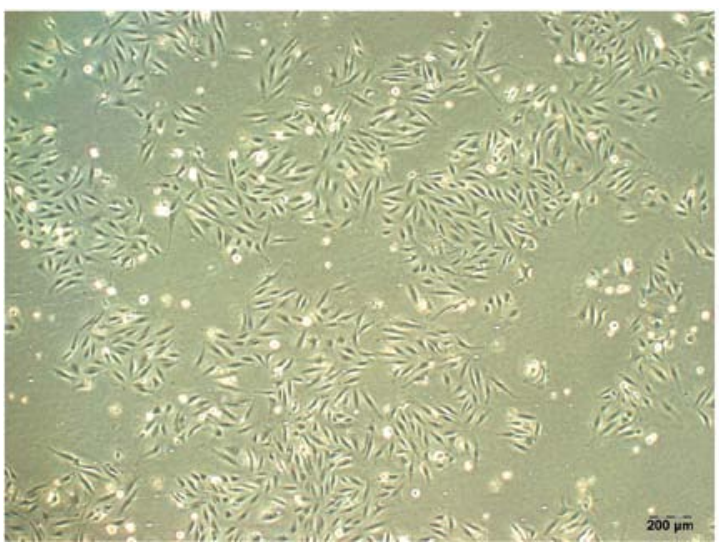

Figure 4. SNU-46-D2 cells.

cells that stably transfected had G418 resistance and continued proliferation. Cells which obtained $\mathrm{G} 418$ resistance continued to culture in the screening of antibiotics environment to make a large amount. Then, several monoclonal cell lines were selected with the limiting dilution method. Three monoclonal cell lines that were infected with retrovirus carrying DJ-1 gene and screened

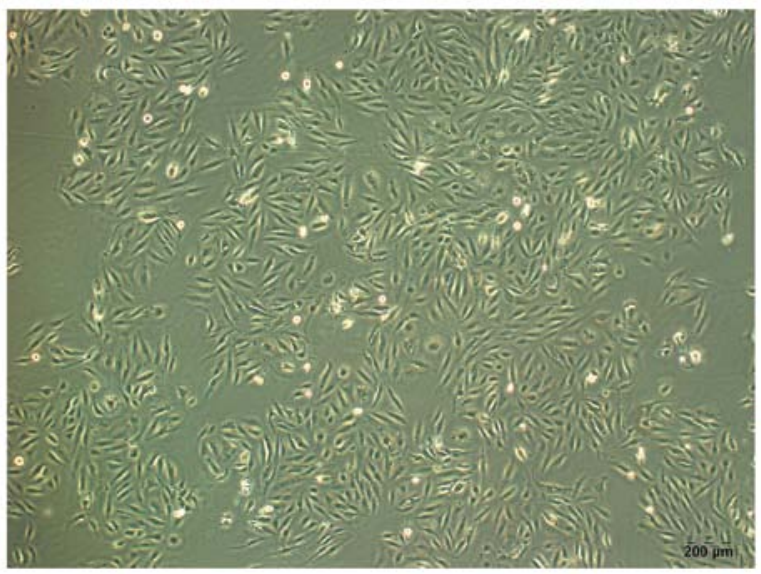

Figure 5. SNU-46-D3 cells.

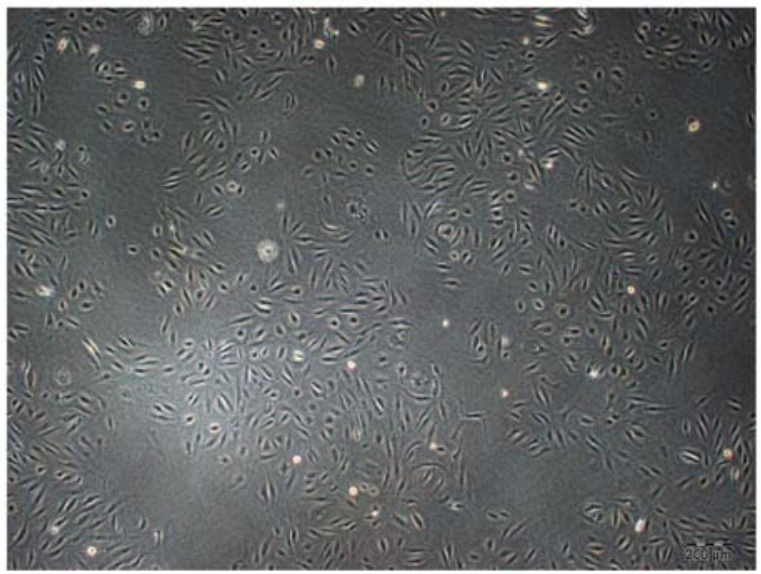

Figure 6. SNU-46-CON cells

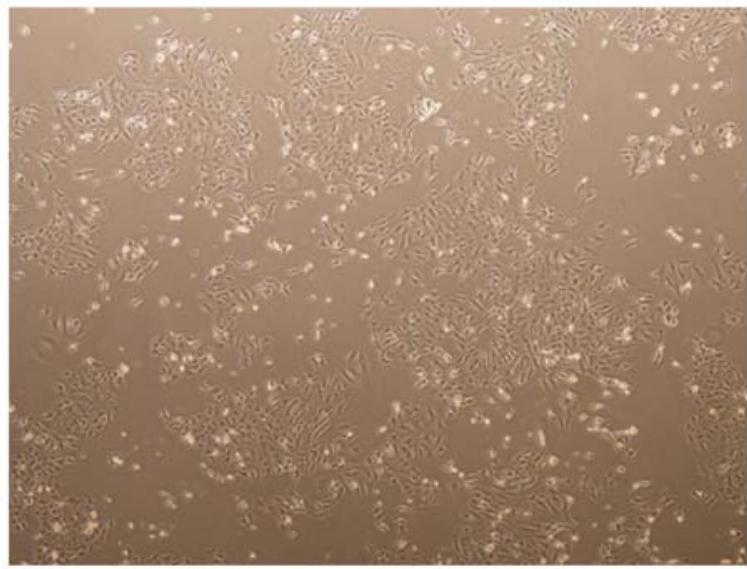

Figure 7. SNU-46 cells.

through G418 were selected and termed SNU-46-D1 (Fig. 3), SNU-46-D2 (Fig. 4) and SNU-46-D3 (Fig. 5). One monoclonal cell line which was infected with empty vector retrovirus not carrying gene DJ-1 and screened after G418 was selected and termed SNU-46-CON (Fig. 6). Fig. 7 shows untreated normal SNU-46 cells. 


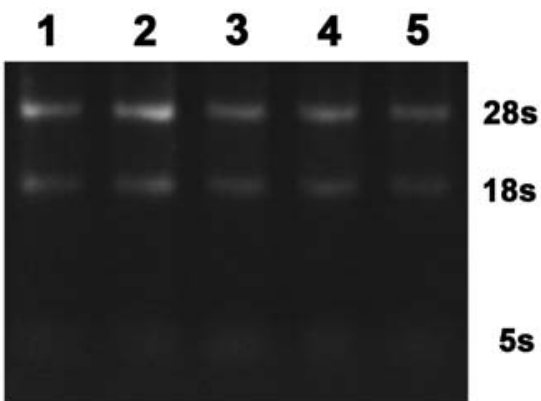

Figure 8. RNA electrophoresis. Lane 1, SNU-46-D1 total RNA; lane 2, SNU-46-D2 total RNA; lane 3, SNU-46-D3 of total RNA; lane 4, SNU-46-CON total RNA; lane 5, SNU-46 total RNA.

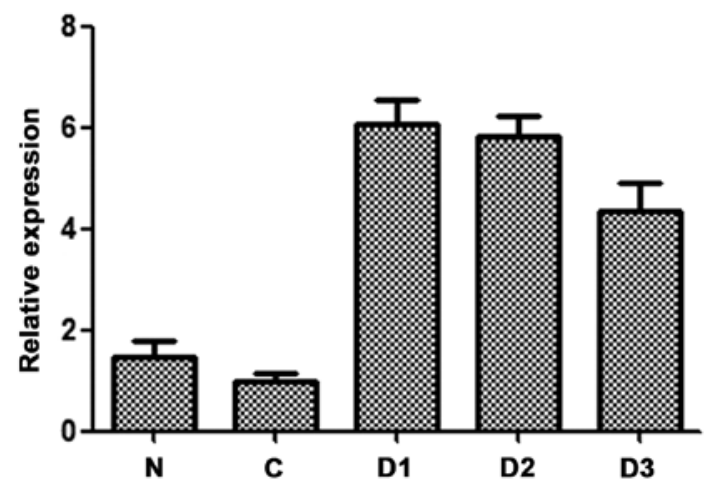

Figure 9. DJ-1 gene expression in five types of cells. N, SNU-46 cells; C, SNU-46-CON cells; D1, SNU-46-D1 cells; D2, SNU-46-D2 cells; D3, SNU-46-D3 cells.

qRT-PCR detection of DJ-1 mRNA expression levels of monoclonal cell lines. RNA sample $(1 \mu \mathrm{l})$ was obtained for $1 \%$ agarose gel electrophoresis $80 \mathrm{~V}$ x $20 \mathrm{~min}$. Total RNA $5 \mathrm{~s}, 18 \mathrm{~s}$ and $28 \mathrm{~s}$ rRNA bands were observed by gel imaging system. Three complete bands proved a complete extraction of total RNA (Fig. 8). DJ-1 gene expression in five types of samples is shown in Fig. 9, and DJ-1 gene expression levels of SNU-46-D1, SNU-46-D2 and SNU-46-D3 are significantly higher than SNU-46-CON and SNU-46 cells.

Western blot detection of DJ-1 protein expression levels in each monoclonal cell line. The total protein was extracted after SNU-46-D1, SNU-46-D2, SNU-46-D3, SNU-46-CON and SNU-46 cell proliferation, and DJ-1 protein expression levels of monoclonal cell lines were detected by western blotting. The results showed that DJ-1 protein expression of SNU-46-D1 cells was significantly higher than other cell groups (Fig. 10). SNU-46-D1 cell protein was extracted again and SNU-46-CON and SNU-46 cells were used as control. Western blotting was repeated twice to verify if DJ-1 protein expression of SNU-46-D1 was higher than normal SNU-46 and SNU-46-CON empty vector control cells (Fig. 11). The results of the three repeats were subjected to grey and statistical analysis, and it showed the relative DJ-1 protein expression of SNU-46-D1 cells was $1.969 \pm 0.137$. There was a significant difference $\left({ }^{*} \mathrm{p}<0.001 ;{ }^{* *} \mathrm{p}<0.001\right.$; Fig. 12) compared to SNU-46 $(0.624 \pm 0.079)$ and SNU-46-CON empty vector

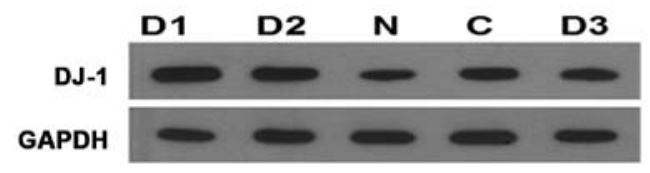

Figure 10. Western blot detection of DJ-1 protein expression levels of 5 cell groups. D1, SNU-46-D1 cells; D2, SNU-46-D2 cells; N, SNU-46 cells; C, SNU-46-CON cells; D3, SNU-46-D3 cells.

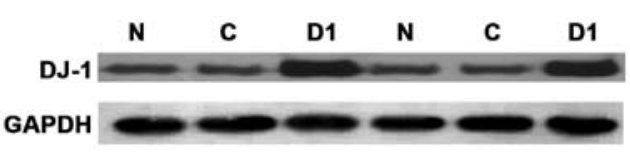

Figure 11. Western blot detection of DJ-1 protein levels of SNU-46-D1 cells. N, SNU-46 cells; C, SNU-46-CON cells; D1, SNU-46-D1 cells.

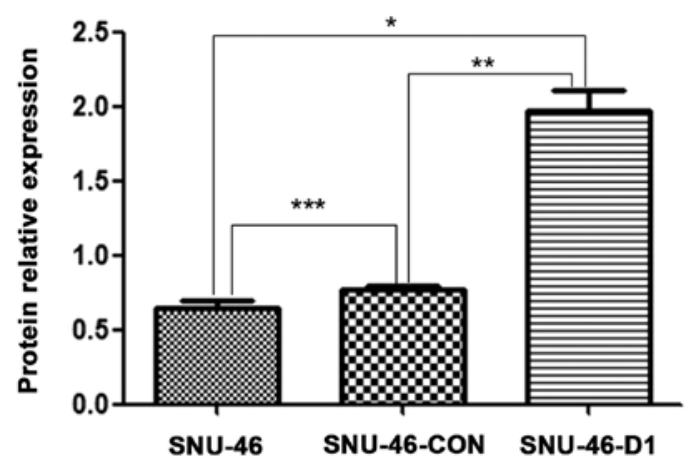

Figure 12. The relative protein expression of three cell lines. " $\mathrm{p}<0.001$; ${ }^{* *} \mathrm{p}<0.001 ;{ }^{* * *} \mathrm{p}=0.081$.

control cells $(0.783 \pm 0.279)$, while the relative DJ-1 protein expression levels of SNU-46 and SNU-46-CON empty vector control cells showed no significant difference $\left({ }^{* * *} \mathrm{p}=0.081\right)$. Compared with the blank control SNU-46 cells, DJ-1 protein expression levels increased 2.16-fold, therefore monoclonal cell line SNU-46-D1 of DJ-1 protein overexpression was successfully constructed.

Overexpression of DJ-1 protein reduces $S N U-46$ cell apoptosis rate. Fig. 13 shows results of the apoptosis rate of three cell lines using flow cytometry with the Annexin V/PI apoptosis kit. There was a significant difference $(\mathrm{p}<0.05)$ of the apoptotic cell rate among SNU-46-D1, SNU-46-CON and SNU-46. Combining multiple comparison results showed the apoptotic ratio of SNU-46-D1 cell line $(3.533 \pm 5.167 \%)$ was significantly lower than that of SNU-46 $(16.397 \pm 5.447 \%)$ and SNU-46-CON cell lines $(16.980 \pm 4.124 \%)(\mathrm{p}=0.019$; $\left.{ }^{* *} \mathrm{p}=0.016\right)$. There was no statistical difference between cell line SNU-46 apoptosis rate and cell line SNU-46-CON $\left.{ }^{* * *} \mathrm{p}=0.890\right)$.

Overexpression of DJ-1 protein promotes $S N U-46$ cell proliferation. Results of CCK-8 detection of cell proliferation are shown in Fig. 14. Forty-eight and $72 \mathrm{~h}$ after inoculation, SNU-46-D1 cell proliferation rate was higher than SNU-46 and SNU-46-CON cells ( $48 \mathrm{~h}, 0.834 \pm 0.336$ vs. $0.676 \pm 0.112$, "p $<0.001 ; 0.834 \pm 0.336$ vs. $0.694 \pm 0.210,{ }^{*} \mathrm{p}<0.001 ; 72 \mathrm{~h}$, 

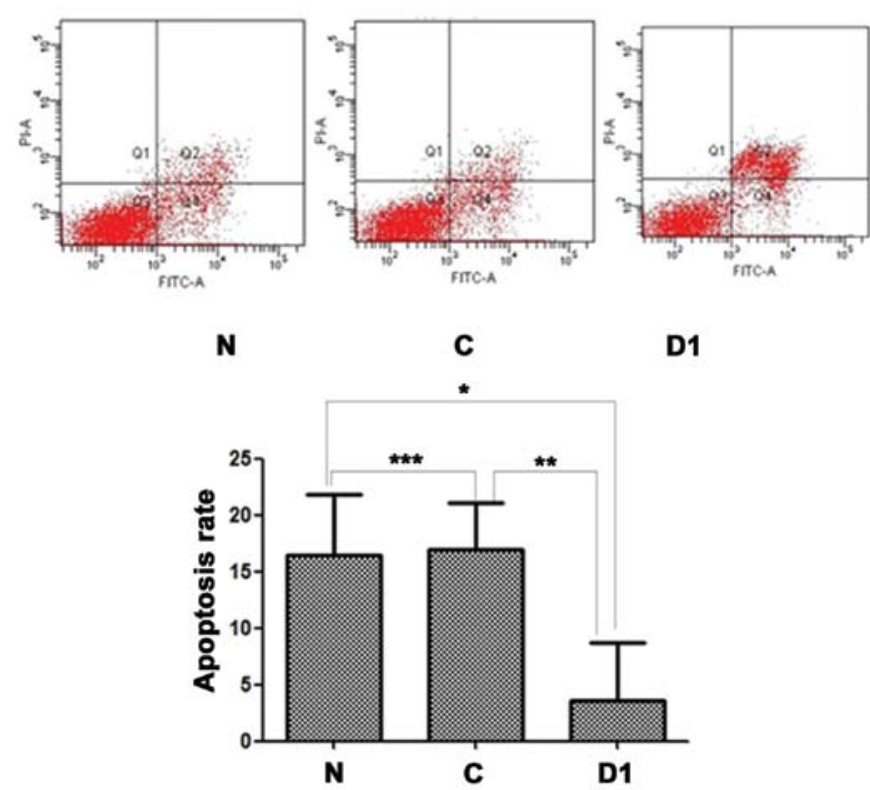

Figure 13. Apoptosis rate of three cell lines. N, SNU-46 cells; C, SNU46-CON cells; D1, SNU-46-D1 cells. ${ }^{*} \mathrm{p}=0.019 ;{ }^{* *} \mathrm{p}=0.016 ;{ }^{* * *} \mathrm{p}=0.890$.

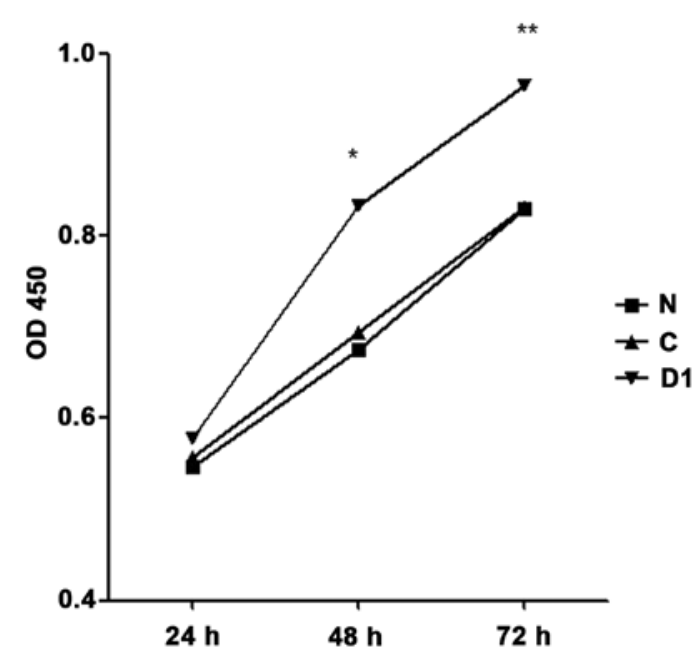

Figure 14. CCK-8 detection of the effect of DJ-1 gene on the proliferation of SNU-46. N, SNU-46 cells; C, SNU-46-CON cells; D1, SNU-46-D1 cells. * $\mathrm{p}<0.001 ; * \mathrm{*}<<0.001$.

$0.965 \pm 0.177$ vs. $0.830 \pm 0.172,{ }^{* *} \mathrm{p}<0.001 ; 0.965 \pm 0.177$ vs. $\left.0.831 \pm 0.180,{ }^{* *} \mathrm{p}<0.001\right)$.

Overexpression of DJ-1 protein enhances $S N U-46$ cell invasion and migration. As shown in Fig. 15, SNU-46-D1 cell invasion force was higher than SNU-46 and SNU-46-CON cells (165.7 \pm 13.6 vs. $100.0 \pm 17.4,{ }^{*} \mathrm{p}=0.001 ; 165.7 \pm 13.6$ vs. $103.0 \pm 7.6$, " $\mathrm{p}=0.001$ ); as shown in Fig. 16, SNU-46-D1 cell migration was higher than SNU-46 and SNU-46-CON cells $(207.3 \pm 13.1$ vs. $175.3 \pm 13.3,{ }^{*} \mathrm{p}=0.036 ; 207.3 \pm 13.1$ vs. $173.0 \pm 12.5,{ }^{*} \mathrm{p}=0.027$ ).

Western blot detection of changes in p-AKT, AKT, p-mTOR, mTOR, PTEN protein expression levels after DJ-1 overexpression. As shown in Figs. 17-19, p-AKT protein expression levels improved after overexpression of DJ-1, while AKT
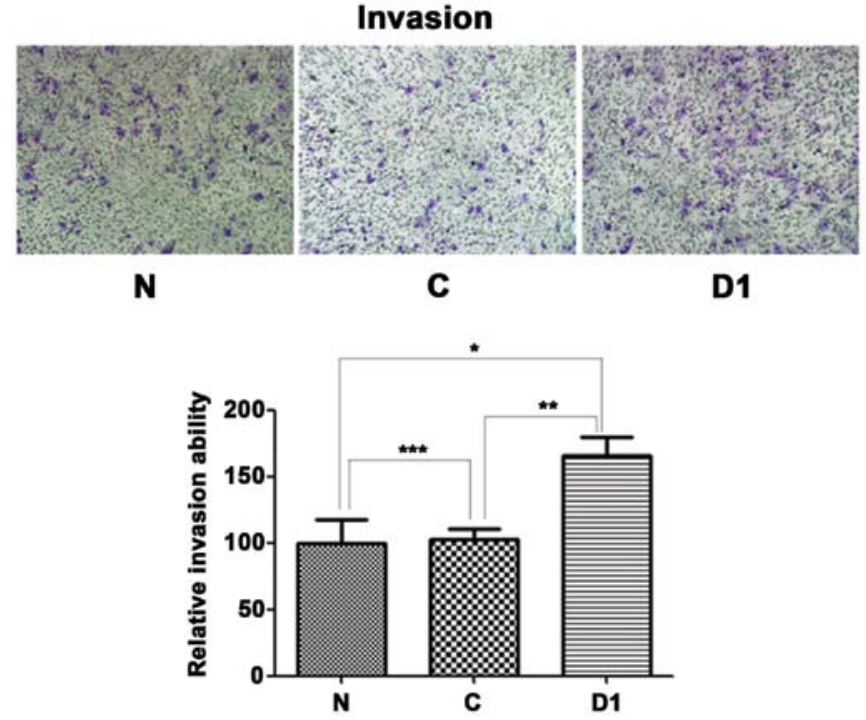

Figure 15. Transwell assay detection of DJ-1 gene impact on the invasion of SNU-46. N, SNU-46 cells; C, SNU-46-CON cells; D1, SNU-46-D1 cells. " $\mathrm{p}=0.001 ;{ }^{* *} \mathrm{p}=0.001 ;{ }^{* * * *} \mathrm{p}=0.794$.

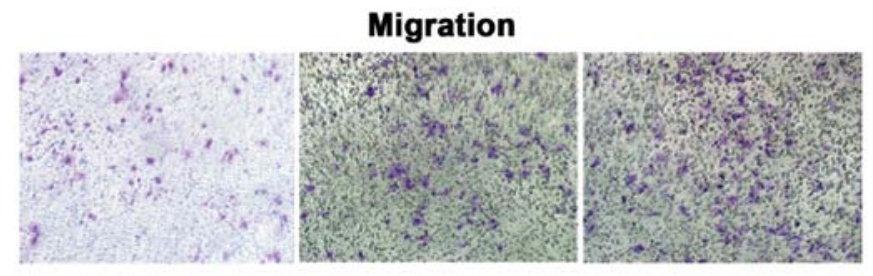

$\mathbf{N}$

C

D1

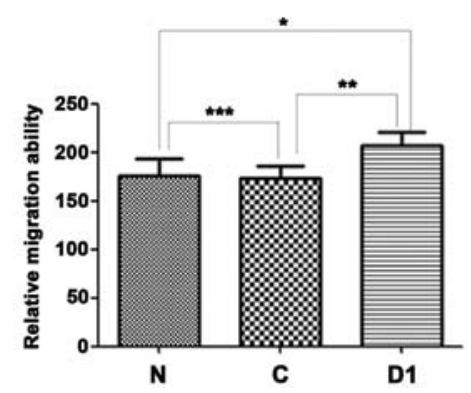

Figure 16. Transwell assay detection of DJ-1 gene impact on migration of SNU-46. N, SNU-46 cells; C, SNU-46-CON cells; D1, SNU-46-D1 cells. * $\mathrm{p}=0.036 ;{ }^{* *} \mathrm{p}=0.027 ;{ }^{* * * *} \mathrm{p}=0.850$

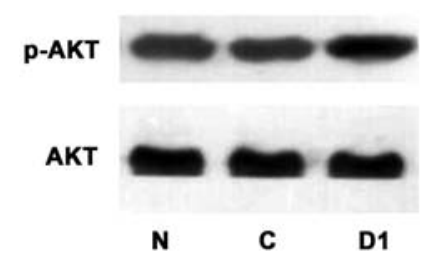

Figure 17. Western blot detection of SNU-46-D1 cells. p-AKT, AKT protein expression levels. N, SNU-46 cells; C, SNU-46-CON cells; D1, SNU-46-D1 cells.

protein expression did not change. p-mTOR protein expression increased and mTOR protein expression did not change. PTEN protein expression decreased. 


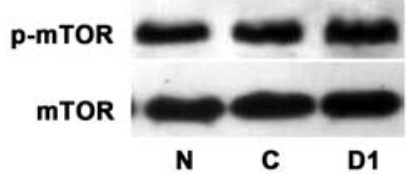

Figure 18. Western blot detection of p-mTOR, mTOR protein levels in SNU-46-D1 cells. N, SNU-46 cells; C, SNU-46-CON cells; D1, SNU-46-D1 cells.

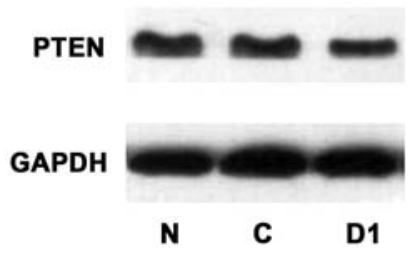

Figure 19. Western blot detection of SNU-46-D1 cells. PTEN protein expression level. N, SNU-46 cells; C, SNU-46-CON cells; D1, SNU-46-D1 cells.

\section{Discussion}

Laryngeal cancer is ranked second in the incidence of head and neck tumors, and the most common histological type is squamous cell carcinoma (13-15). Currently, the overall 5-year survival rate is still hovering at $55-65 \%(16-21)$. The molecular biological mechanisms of the pathogenesis of laryngeal cancer remain to be explored. Previous studies found that there was overexpression of DJ-1 of laryngeal tissue and Hep-2 cells, and poorly differentiated laryngeal cancer had higher DJ-1 expression level than well differentiated laryngeal cancer. Silencing DJ-1 gene can effectively inhibit the proliferation of Hep-2 cells, inducing apoptosis (22). However, the effect of DJ-1 gene overexpression in laryngeal cancer cells on the biological behavior of laryngeal tumor cells has not been reported. Also, the effect of DJ-1 gene overexpression on laryngeal cancer proliferation, migration, invasion, apoptosis and other biological characteristics of the tumor cells remains unclear. The signaling pathway through which the DJ-1 gene mediates to affect laryngeal cancer is also unknown. Therefore, on the basis of previous studies, we stably transfected DJ-1 gene through retrovirus to laryngeal squamous cell carcinoma SNU-46 cell line and successfully screened out laryngeal squamous cell lines with stable overexpression of DJ-1 protein. The differences of biological characteristics between laryngeal carcinoma cell line SNU-46-D1 with DJ-1 gene overexpression and normal laryngeal cancer cells was detected, thereby we preliminarily studied the role of DJ-1 overexpression in cell proliferation, invasion, migration, apoptosis, and established a research basis for future studies in nude mice.

The early pre-experiment showed that the stable transfection efficiency of SNU-46 laryngeal squamous cell line with electroporation and lipofection is very low. In the present study, retrovirus pLNCX2-DJ-1 vector carrying DJ-1 gene was constructed; pVSV-G packaging plasmids and GP2-293 packaging cells were used to successfully package viral particles carrying DJ-1 gene, which in turn infected SNU-46 cells to achieve stable DJ-1 gene expression in SNU-46 laryngeal cancer cells. Since the pLNCX2-DJ-1 vector also contains the neo gene in addition to DJ-1 gene sequence, successfully transfected cells obtained resistance to antibiotic G418. Therefore, cells that were not successfully transfected could be eliminated through G418 screening. In the present study, a laryngeal carcinoma cell line with stable overexpression of DJ-1 protein was successfully screened out, SNU-46-D1. After western blotting, DJ-1 protein expression increased 2.16fold compared to normal SNU-46 cells. We also screened an SNU-46 cell line as the empty vector control (SNU-46-CON), of which DJ-1 expression level was no different to untreated cells.

Apoptosis is a suicide mechanism of cell active participation (22) and evasion apoptosis is one of the basic characteristics of tumor cells. The defect of apoptotic signaling pathway is widespread in cancer. In the present study, after flow cytometry detection with Annexin V/PI apoptosis kit, the results showed that the apoptosis rate of SNU-46-D1 was significantly lower than the rates of cell lines SNU-46 and SNU-46-CON, suggesting that overexpression of the DJ-1 gene inhibited apoptosis of laryngeal cancer cells. Previous reports showed that DJ-1 gene silencing can induce apoptosis of laryngeal cancer Hep-2 cells (23). Therefore, the DJ-1 gene may play a role in inhibiting apoptosis of laryngeal cancer cells to prolong laryngeal cell survival time and provide the conditions for the growth of laryngeal cancer cells. CCK- 8 detection of cell proliferation showed that SNU-46-D1 cell proliferation rate was faster than normal SNU-46 cells. The difference was statistically significant. This suggests that the DJ-1 gene plays a facilitating role in the proliferation of SNU-46 cells. After detecting the invasion and migration ability of SNU-46 laryngeal cancer cells and SNU-46-D1 cells with stably transfected DJ-1 protein overexpression by Transwell chamber experiments, we found that after stable transfection by up to $2 \mathrm{x}$ the DJ-1 protein expression, invasion of SNU-46-D1 cells increased by $\sim 65 \%$ compared to normal SNU- 46 , and migration increased by $\sim 20 \%$. This suggests that overexpression of DJ-1 protein can enhance the ability of laryngeal cancer cell invasion and migration. Through stable transfection for laryngeal cells SNU-46 to overexpress DJ-1 protein, the apoptosis rate decreased, proliferation rate increased, invasion and migration capabilities also improved. This suggests that the DJ-1 gene may play a role in laryngeal cancer genes similar to oncogenes, and laryngeal DJ-1 gene high expression may lead to increased laryngeal malignancy, reducing the prognosis. Zhu et al used immunohistochemistry to detect tumor tissues of 71 postoperative patients with laryngeal squamous cell carcinoma and laryngeal mucosa of 9 patients with non-laryngeal who were treated between January 1995 and January 2006. After analyzing the relationship between DJ-1 protein expression and clinicopathological features, they found DJ-1 protein expression levels in laryngeal squamous cell carcinoma were higher than in normal laryngeal mucosa, and survival rates may be lower for laryngeal cancer patients with higher levels of DJ-1 protein expression (23). In 2012, Zhu et al also found that the DJ-1 protein overexpression in tumor tissue of supraglottic laryngeal squamous cell carcinoma was associated with lymph node metastasis. Lymph node metastasis has higher incidence for patients with high DJ-1 protein expression. The five-year survival rate of patients with high DJ-1 protein expression in supraglottic laryngeal squamous cell carcinoma 
is also lower (53.9 vs. $88.0 \%$; $\mathrm{p}=0.007$; log-rank test) (24). The data on DJ-1 expression levels and clinical tumor malignancy and prognosis are consistent with our conclusion regarding the impact of DJ-1 protein on laryngeal carcinoma cell SNU-46 functions. The DJ-1 gene may play a role similar to oncogenes in laryngeal carcinoma, and can inhibit apoptosis. The DJ-1 gene can extend the survival time of laryngeal cancer cells, and provide conditions for laryngeal cancer cell growth. At the same time, the DJ-1 gene can promote the proliferation of laryngeal cancer cells, resulting in the rapid expansion of laryngeal cancer cells and accelerated tumor growth. More importantly, the DJ-1 gene can enhance the ability of laryngeal cancer cell invasion and migration. It may lead to tumor lymph node metastasis and distant metastasis, resulting in an adverse impact on the prognosis of patients.

Through stable transfection of DJ-1 gene to SNU-46 cells and detection of changes in the biological characteristics of the tumor cells, this study preliminarily showed the DJ-1 gene can inhibit tumor cell apoptosis and promote proliferation, migration and invasion of tumor cells. Regarding the molecular pathway mechanism which leads to changes in the biological characteristics of these tumors, current studies support that DJ-1 protein affects biological properties of the cells mainly through negative regulation of PTEN (phosphatase and tensin homolog) expression. Kim et al found DJ-1 expression in breast cancer specimens was negatively correlated with PTEN and positively correlated with $\mathrm{PKB} / \mathrm{Akt}$ (6). Zhu et al found that the DJ-1 protein overexpression in tumor tissue of supraglottic laryngeal squamous cell carcinoma was accompanied by low expression of PTEN (24). PTEN is a tumor suppressor that can inhibit the phosphorylation of PIP2 to reduce the PIP3 generation and ultimately reduce PKB/Akt activation and inhibit cell growth (4.) DJ-1 protein overexpression can lead to reduced expression of PTEN, thereby removing the inhibition of PTEN on cell growth, and promoting cell growth and proliferation. Studies have also suggested that the DJ-1 gene can promote the PI3K/AKT/mTOR pathway in lung cancer cells by regulating PTEN gene, thereby enabling increased expression of a variety of detoxifying enzymes and promoting cell proliferation (11). Regarding the approach of DJ-1 gene regulation on PTEN, some researchers believe the DJ-1 gene may be involved in the regulation of PTEN gene through the PI3K-AKT/PKB signaling pathway to act on c-myc (25). Other researchers consider that DJ-1 may regulate PTEN through their redoxsensitive molecular chaperone activity (26). In addition, studies have also found that DJ-1 can activate hypoxia-inducible factor 1 (HIF1) in the absence of oxygen, and has a crucial role in maintaining the stability of HIF1, therefore DJ-1 is resistant to hypoxia-mediated apoptosis, protecting cells under hypoxia. Meanwhile DJ-1 can also regulate activity of AMP protein kinase (AMPK) which controls cell metabolism. This regulation is more obvious under a hypoxic environment (27). These studies suggest that the laryngeal apoptosis rate decreased after the overexpression of DJ-1 most likely since DJ-1 can help tumor cells to evade apoptosis incurred by an anoxic environment.

Our previous studies showed Akt and mTOR overexpression in laryngeal cancer are correlated with prognosis, and are negatively correlated with tumor differentiation. The recurrent rate of patients with overexpression of downstream gene EIF4E on the signaling pathway in laryngeal tissue and overexpression of eIF4E on surgical margins is high, and the prognosis is poor. mTOR inhibitor rapamycin can stop Hep-2 cells in the G1 phase, inhibiting their proliferation and promoting apoptosis. Joint application of rapamycin and cisplatin can have a synergistic effect (28). LSCC DJ-1 protein overexpression and Akt/mTOR overexpression often occur simultaneously. Previous studies have shown DJ-1 may mediate the PI3K/AKt/mTOR signaling pathway to affect the survival of a variety of tumor cells $(6,27)$. Through the construction of SNU-46 laryngeal squamous cell line of stably overexpressed DJ-1 protein, and detection of expression level changes of proteins associated with the PI3K/AKt $/ \mathrm{mTOR}$ signaling pathway between this cell and normal SNU-46 cell line, this study provided preliminary validation that DJ-1 may affect the biological characteristics of laryngeal cancer cells through the PI3K/AKt/mTOR signaling pathway.

Through the construction of stable overexpression of DJ-1 protein in laryngeal carcinoma cell lines, the present study established a foundation for subsequent in vivo experiments in nude mice. We will carry out more comprehensive studies on SNU-46-D1 cell lines with stable overexpression of the DJ-1 gene to gain more in-depth understanding of the function of DJ-1 gene in the occurrence and development of laryngeal cancer and cell signaling pathways mediated by DJ-1 gene, thereby laying the foundations for the assessment of the feasibility and efficacy of the DJ-1 gene as a new therapeutic target for laryngeal cancer.

\section{Acknowledgements}

This study was supported by the National Natural Science Foundation of China (81072224), the Young Faculty Cultivation Project of Sun Yat-sen University (10ykpy10), and the Science and Technology Planning Project of Guangdong Province (2009B030801109).

\section{References}

1. Nagakubo D, Taira T, Kitaura H, et al: DJ-1, a novel oncogene which transforms mouse NIH3T3 cells in cooperation with ras. Biochem Biophys Res Commun 231: 509-513, 1997.

2. Annesi G, Savettieri G, Pugliese P, et al: DJ-1 mutations and parkinsonism-dementia-amyotrophic lateral sclerosis complex. Ann Neurol 58: 803-807, 2005.

3. Miller DW, Ahmad R, Hague S, et al: L166P mutant DJ-1, causative for recessive Parkinson's disease, is degraded through the ubiquitin-proteasome system. J Biol Chem 278: 36588-36595, 2003.

4. Martinat C, Shendelman S, Jonason A, et al: Sensitivity to oxidative stress in DJ-1-deficient dopamine neurons: an ES-derived cell model of primary Parkinsonism. PLoS Biol 2: e327, 2004

5. Moore DJ, Zhang L, Troncoso J, et al: Association of DJ-1 and parkin mediated by pathogenic DJ-1 mutations and oxidative stress. Hum Mol Genet 14: 71-84, 2005.

6. Kim RH, Peters M, Jang Y, et al: DJ-1, a novel regulator of the tumor suppressor PTEN. Cancer Cell 7: 263-273, 2005.

7. MacKeigan JP, Clements CM, Lich JD, et al: Proteomic profiling drug-induced apoptosis in non-small cell lung carcinoma: identification of RS/DJ-1 and RhoGDIa. Cancer Res 63: 6928-6934, 2003.

8. Hod Y: Differential control of apoptosis by DJ-1 in prostate benign and cancer cells. J Cell Biochem 92: 1221-1233, 2004

9. Le Naour F, Misek DE, Krause MC, et al: Proteomics-based identification of RS/DJ-1 as a novel circulating tumor antigen in breast cancer. Clin Cancer Res 7: 3328-3335, 2001. 
10. Yoshino T, Shiina H, Urakami S, et al: $\mathrm{Bcl}-2$ expression as a predictive marker of hormone-refractory prostate cancer treated with taxane-based chemotherapy. Clin Cancer Res 12: 6116-6124, 2006.

11. Shi W, Zhang X, Pintilie M, et al: Dysregulated PTEN-PKB and negative receptor status in human breast cancer. Int J Cancer 104: 195-203, 2003.

12. Davidson B, Hadar R, Schlossberg A, et al: Expression and clinical role of DJ-1, a negative regulator of PTEN, in ovarian carcinoma. Hum Pathol 39: 87-95, 2008.

13. Chu EA and Kim YJ: Laryngeal cancer: diagnosis and preoperative work-up. Otolaryngol Clin North Am 41: 673-695, 2008.

14. Parkin DM, Bray F, Ferlay J and Pisani P: Global cancer statistics, 2002. CA Cancer J Clin 55: 74-108, 2005.

15. Adelstein DJ, Li Y, Adams GL, et al: An intergroup phase III comparison of standard radiation therapy and two schedules of concurrent chemoradiotherapy in patients with unresectable squamous cell head and neck cancer. J Clin Oncol 21: 92-98, 2003.

16. Hoffman HT, Porter K, Karnell LH, et al: Laryngeal cancer in the United States: changes in demographics, patterns of care, and survival. Laryngoscope 116: 1-13, 2006.

17. Denis F, Garaud P, Bardet E, et al: Final results of the 94-01 French Head and Neck Oncology and Radiotherapy Group randomized trial comparing radiotherapy alone with concomitant radiochemotherapy in advanced-stage oropharynx carcinoma. J Clin Oncol 22: 69-76, 2004.

18. Forastiere AA, Maor M, Weber RS, et al: Long-term results of intergroup RTOG 91-11: a phase III trial to preserve the larynxinduction cisplatin/5-FU and radiation therapy versus concurrent cisplatin and radiation therapy versus radiation therapy. J Clin Oncol 24 (Suppl 18): 5517, 2006.

19. Seiwert TY and Cohen EE: State-of-the-art management of locally advanced head and neck cancer. Br J Cancer 92: 1341-1348, 2005.
20. Brockstein B, Haraf DJ, Rademaker AW, et al: Patterns of failure, prognostic factors and survival in locoregionally advanced head and neck cancer treated with concomitant chemoradiotherapy: a 9-year, 337-patient, multi-institutional experience. Ann Oncol 15: 1179-1186, 2004.

21. Kutter J, Ozsahin M, Monnier P and Stupp R: Combined modality treatment with full-dose chemotherapy and concomitant boost radiotherapy for advanced head and neck carcinoma. Eur Arch Otorhinolaryngol 262: 1-7, 2005.

22. Kerr JF, Wyllie AH and Currie AR: Apoptosis: a basic biological phenomenon with wide-ranging implications in tissue kinetics. Br J Cancer 26: 239-257, 1972.

23. Zhu XL, Wang ZF, Lei WB, et al: DJ-1: a novel independent prognostic marker for survival in glottic squamous cell carcinoma. Cancer Sci 101: 1320-1325, 2010.

24. Zhu XL, Wang ZF, Lei WB, et al: Tumorigenesis role and clinical significance of DJ-1, a negative regulator of PTEN, in supraglottic squamous cell carcinoma. J Exp Clin Cancer Res 31: 94, 2012.

25. Sitaram RT, Cairney CJ, Grabowski P, et al: The PTEN regulator DJ-1 is associated with hTERT expression in clear cell renal cell carcinoma. Int J Cancer 125: 783-790, 2009.

26. Shendelman S, Jonason A, Martinat C, et al: DJ-1 is a redoxdependent molecular chaperone that inhibits $\alpha$-synuclein aggregate formation. PLoS Biol 2: e362, 2004.

27. Vasseur S, Afzal S, Tardivel-Lacombe J, et al: DJ-1/PARK7 is an important mediator of hypoxia-induced cellular responses. Proc Natl Acad Sci USA 106: 1111-1116, 2009.

28. Lei W, Jia T, Su Z, et al: Combined effect of rapamycin and cisplatin on survival of Hep-2 cells in vitro. Oncol Res 18: 73-81, 2009. 